\title{
In Vitro Antimicrobial Activity of Fosfomycin, Vancomycin and Daptomycin Alone, and in Combination, Against Linezolid-Resistant Enterococcus faecalis
}

Wei Yu · Jiajie Zhang · Jiepeng Tong $\cdot$ Li Zhang $\cdot$ Yaqiong Zhan •

Yicheng Huang • Yunqing Qiu

Received: August 7, 2020 / Accepted: September 10, 2020 / Published online: September 22, 2020

(C) The Author(s) 2020

\begin{abstract}
Introduction: Enterococcus faecalis is a significant cause of nosocomial infections and is difficult to treat because of intrinsic and acquired resistance to many antibiotics. In addition, the emergence of linezolid-resistant $E$. faecalis (LZREfa) is reducing the choices available for antiinfective therapy. The aim of this study was to examine the in vitro antibacterial effects of fosfomycin (FM), vancomycin (VAN) and daptomycin (DAP), alone and in combination, against LZR-Efa.
\end{abstract}

Digital Features To view digital features for this article go to https://doi.org/10.6084/m9.figshare.12928559.

Electronic supplementary material The online version of this article (https://doi.org/10.1007/s40121020-00342-1) contains supplementary material, which is available to authorized users.

W. Yu · J. Tong $\cdot$ L. Zhang $\cdot$ Y. Zhan $\cdot$ Y. Qiu (ه) State Key Laboratory for Diagnosis and Treatment of Infectious Diseases, National Clinical Research Center for Infectious Diseases, Collaborative Innovation Center for Diagnosis and Treatment of Infectious Diseases, Zhejiang Provincial Key Laboratory for Drug Clinical Research and Evaluation, The First Affiliated Hospital, Zhejiang University School of Medicine, Hangzhou, China e-mail: qiuyq@zju.edu.cn

\section{J. Zhang · Y. Huang}

Department of Infectious Diseases, Zhejiang Provincial People's Hospital, People's Hospital of Hangzhou Medical College, Hangzhou, China
Methods: Five LZR-Efa strains and E. faecalis ATCC 29212 were studied. The antibacterial effects of FM, and of FM, VAN and DAP, were assessed using the time-kill assay. Biofilm formation and elimination were evaluated by crystal violet staining.

Results: When used at concentrations greater than $0.5 \times$ MIC, FM did not produce dose-dependent effects against LZR-Efa isolates. The use of DAP (47.1 mg/L) alone, and FM $(83 \mathrm{mg} / \mathrm{L})$ combined with DAP $(20.6 \mathrm{mg} / \mathrm{L})$, produced a persistent inhibitory effect against both planktonic LZR-Efa isolates and those forming biofilms. In addition, FM and VAN combined with glucose-6-phosphate produced visible eradication effects against biofilms grown for $24 \mathrm{~h}$, while DAP alone or combined with FM resulted in the best eradication activity against biofilms grown for $72 \mathrm{~h}$ prior to exposure.

Conclusion: The use of FM combined with DAP provided the best potential therapeutic option for treating LZR-Efa infections out of those tested. In addition, the optimum treatment for biofilm elimination depended on the stage of biofilm formation.

Keywords: Biofilm; Combination therapy; Daptomycin; Fosfomycin; Vancomycin 


\section{Key Summary Points}

The pattern of fosfomycin activity against linezolid-resistant Enterococcus faecalis (LZR-Efa) was not concentrationdependent.

The killing effect of DAP monotherapy was much more pronounced than that of FM and VAN.

DAP (47.1 mg/L) monotherapy and FM ( $83 \mathrm{mg} / \mathrm{L})$ combined with DAP $(20.6 \mathrm{mg} /$ L) had a persistent effect on planktonic LZR-Efa and biofilm formation.

FM combined with VAN effectively eliminated newly formed biofilm, while FM combined with DAP was better against mature biofilm.

\section{DIGITAL FEATURES}

This article is published with digital features to facilitate understanding of the article. To view digital features for this article go to https://doi. org/10.6084/m9.figshare.12928559.

\section{INTRODUCTION}

Enterococcus faecalis is a significant opportunistic pathogen and is especially noted for causing nosocomial infections. To date, linezolid has been an effective antibiotic for use against multidrug-resistant (MDR) Gram-positive bacteria, including vancomycin-resistant Enterococcus (VRE). However, the increasing occurrence of linezolid-resistant $E$. faecalis (LZREfa) has attracted global attention [1]. In addition, the therapeutic choices for treating MDR enterococci were limited because of intrinsic and acquired resistance to many antibiotics [2]. Resistance toward linezolid is mainly mediated by the acquisition of $c$ fr or optrA genes, mutations in domain $\mathrm{V}$ of the 23S rRNA, and L3/L4 ribosomal proteins [3]. It is of note that the emergence of LZR-Efa had no direct connection with prior linezolid exposure [4].

Numerous studies have demonstrated that biofilm formation leads to low-level linezolid resistance [5]. Biofilm formation makes infections more difficult to treat because of decreased diffusion and penetration of antibiotics [6]. Previous research has shown that fosfomycin (FM) and daptomycin (DAP) were active against both planktonic and adherent $E$. faecalis isolates $[7,8]$. However, the choice of antibiotics against enterococcal biofilms is limited, especially for those formed by LZR-Efa. Thus, to elucidate in vitro effectiveness, we compared the antibacterial effects of FM, vancomycin (VAN) and DAP against planktonic LZR-Efa and their biofilms.

\section{METHODS}

\section{Bacterial Strains and Antimicrobial Agents}

Five LZR-Efa strains (8714, 13470, 19663, 24393,31890 ) were isolated from the blood or urine of patients hospitalised at the First Affiliated Hospital of Zhejiang University (Hangzhou, China). The reference strain ATCC 29212 was also used. The antimicrobial susceptibility and genetic characteristics of the five LZR-Efa strains were described in our previous study [3].

The antimicrobial agents FM, VAN and DAP were purchased from Dalian Meilun Biotech (Dalian, China). Glucose-6-phosphate (G6P) was obtained from Sigma-Aldrich (St Louis, MO, USA). Media were supplemented with $50 \mathrm{mg} / \mathrm{L}$ $\mathrm{Ca}^{2+}$ in experiments using DAP according to Clinical and Laboratory Standards Institute (CLSI) recommendations [9].

\section{Antibacterial Activity of FM}

The effects of FM against three LZR-Efa isolates $(8714,24393,31890)$ and ATCC 29212 were determined using twofold increasing concentrations $(0.5 \times \mathrm{MIC}$ to $16 \times \mathrm{MIC})$ in Mueller-Hinton broth (MHB) containing $25 \mathrm{mg} / \mathrm{L}$ G6P [9]. 


\section{Time-Kill Assays}

The bactericidal activities of FM, VAN and DAP, alone or in combination, against the five LZREfa isolates and ATCC 29212 were investigated using the time-kill method [10]. The following concentrations were used: FM $83 \mathrm{mg} / \mathrm{L}$ [11]; VAN 13.3 and $6 \mathrm{mg} / \mathrm{L}$ [12]; DAP, 20.6, 31.1 and $47.1 \mathrm{mg} / \mathrm{L}$ [13]; FM $83 \mathrm{mg} / \mathrm{L}+$ VAN $13.3 \mathrm{mg} / \mathrm{L}$; $\mathrm{FM} 83 \mathrm{mg} / \mathrm{L}+\mathrm{VAN} 6 \mathrm{mg} / \mathrm{L}$; and $\mathrm{FM} 83 \mathrm{mg} /$ $\mathrm{L}+\mathrm{DAP} 20.6 \mathrm{mg} / \mathrm{L}$. The media were supplemented with $25 \mathrm{mg} / \mathrm{L} \mathrm{G6P}$ for testing of FM.

\section{Anti-Biofilm Formation Testing}

The five LZR-Efa isolates and ATCC 29212 were inoculated into 96-well polystyrene microtiter plates containing MHB and different FM, VAN and DAP concentrations, and incubated for $24 \mathrm{~h}, 48 \mathrm{~h}$ and $72 \mathrm{~h}$. The medium was either supplemented with $25 \mathrm{mg} / \mathrm{L}$ G6P or not. After static incubation, plates were washed with $0.9 \%$ saline to remove unbound bacteria, stained with $1 \%$ crystal violet $(\mathrm{CV})$ for $30 \mathrm{~min}$, and the $\mathrm{CV}$ was then dissolved in absolute alcohol added to the wells. The absorbance of each well was quantified using a plate reader at $590 \mathrm{~nm}$.

\section{Biofilm Elimination Assay}

Six isolates were grown for $24 \mathrm{~h}, 48 \mathrm{~h}$ and $72 \mathrm{~h}$ in $\mathrm{MHB}$, and the supernatant was carefully removed. The established biofilms were washed twice with $200 \mu \mathrm{L}$ sterile normal saline, and $200 \mu \mathrm{L}$ volumes of different concentrations of FM, VAN and DAP were added, with incubation continued for a further $24 \mathrm{~h}$ and $48 \mathrm{~h}$ to assess biofilm-eradicating effects. Biofilm formation was measured as described above.

\section{Statistical Analysis}

Data of biofilm formation were compared using the Wilcoxon rank-sum test. $P$ values less than 0.05 were considered to be statistically significant. Data analysis was used SPSS 23.0 for Windows (SPSS Inc., Chicago, IL, USA).
This study was approved by the Ethics Committee of the First Affiliated Hospital, College of Medicine, Zhejiang University with written informed consent from all subjects (Reference Number 2018502).

\section{RESULTS}

\section{Antibacterial Activity of FM}

The addition of $\mathrm{FM}$ at a concentration of $0.5 \times$ MIC had no obvious bactericidal effect against three LZR-Efa isolates $(8714,24393$, 31890) and ATCC 29212 (Fig. 1). Increasing the concentration from $1 \times$ MIC to $16 \times$ MIC did not yield increased antimicrobial effects, indicating that the activity of FM against LZR-Efa was not concentration-dependent at concentrations greater than $1 \times$ MIC.

\section{Bacterial Time-Kill Effect}

The growth and inhibition curves for ATCC 29212 and five clinical LZR-Efa isolates exposed to antibiotics at average steady-state serum concentrations are shown in Fig. 2. The killing effect of DAP monotherapy was greater than that for FM and VAN. VAN present at $13.3 \mathrm{mg} / \mathrm{L}$ did not produce a better bactericidal effect than VAN added at less than half this concentration $(6 \mathrm{mg} / \mathrm{L})$. In addition, FM combined with VAN did not show a significant synergistic effect compared with the use of either antimicrobial alone. It is noteworthy that FM $(83 \mathrm{mg} / \mathrm{L})$ combined with DAP $(20.6 \mathrm{mg} / \mathrm{L})$ and DAP monotherapy (47.1 mg/L) showed persistent bactericidal activity against all six isolates without re-growth at $24 \mathrm{~h}$.

\section{Anti-Biofilm Formation and Biofilm Elimination Activities}

With the exception of isolate 13470, five other isolates produced strong biofilms (Fig. 3).

There was a significant difference of antibiofilm formation activity among nine antibiotic regimens and control $(P \leq 0.007)$ (Supplementary Table 1$)$. DAP monotherapy inhibited 
(a)
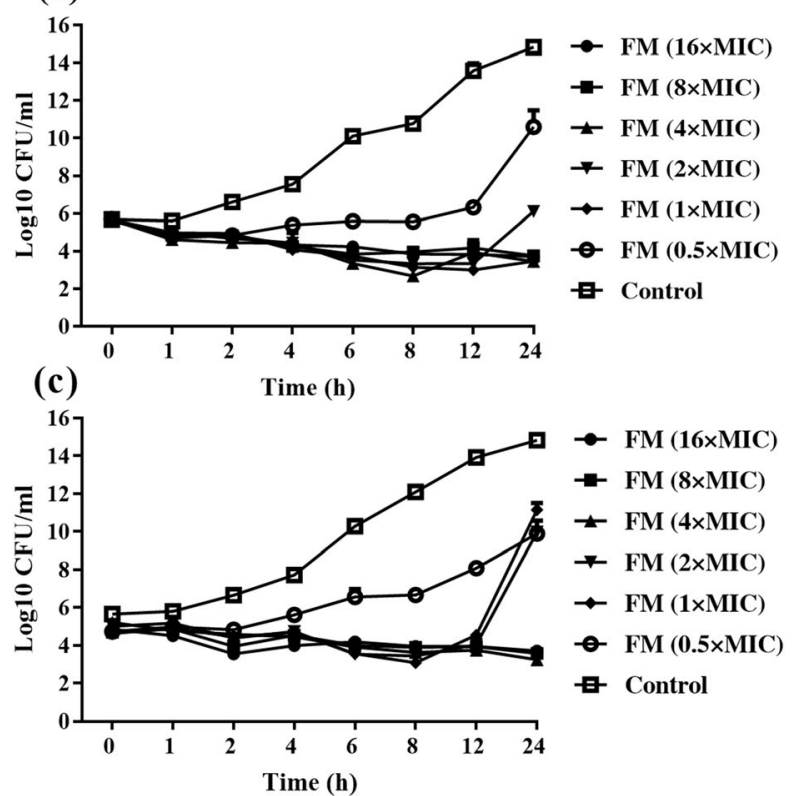

(b)
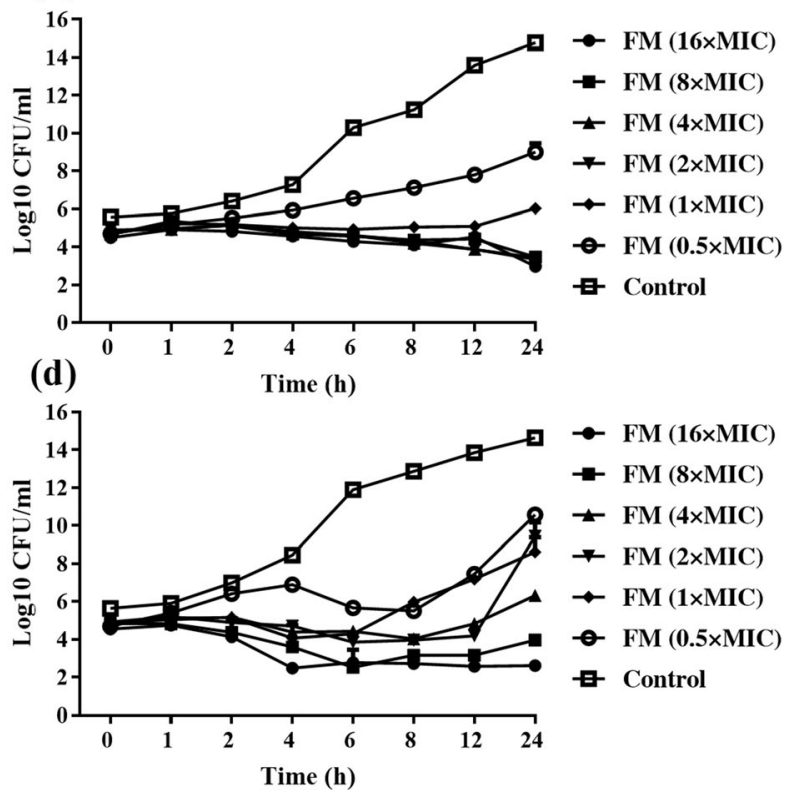

Fig. 1 Antibacterial activity of fosfomycin against ATCC 29212 and three LZR-Efa isolates. a ATCC 29212; b 8714; c 24393; d 31890
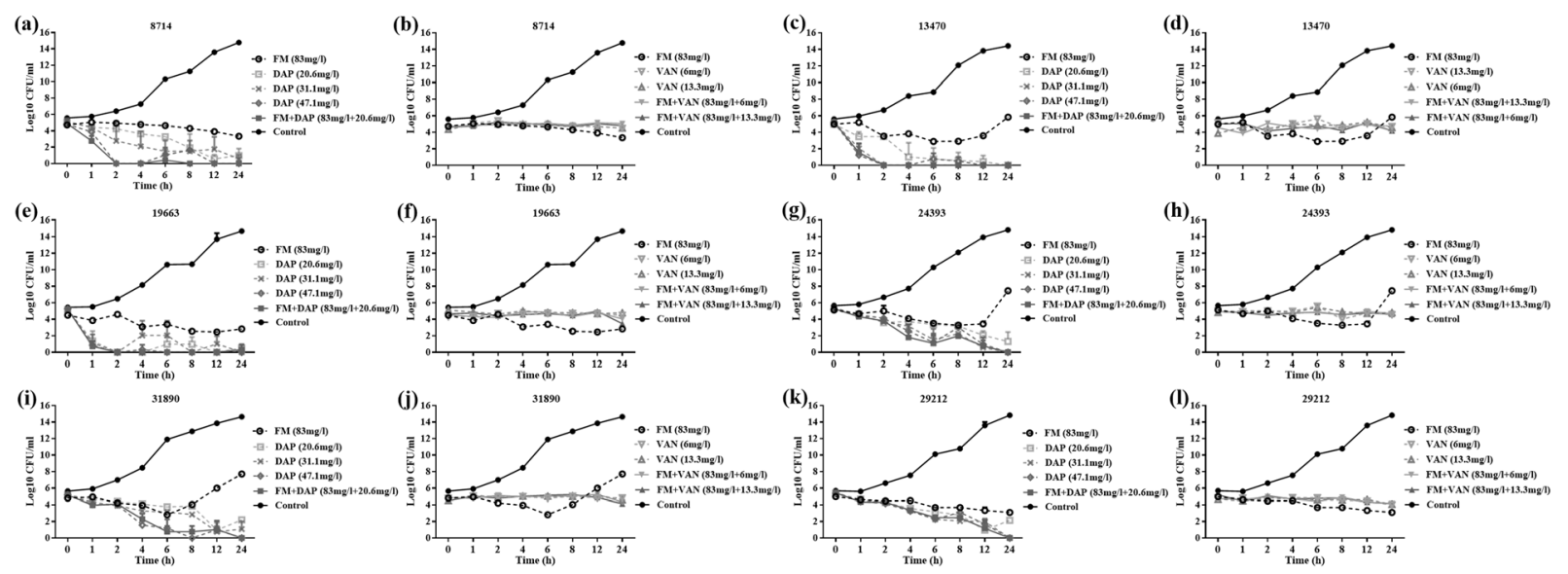

Fig. 2 In vitro time-kill assays of fosfomycin (FM), vancomycin (VAN) and daptomycin (DAP) monotherapy and in combination against ATCC 29212 and five LZR-

LZR-Efa biofilm formation to a greater extent than FM or VAN monotherapies did. The presence of G6P promoted the formation of biofilm in the absence of antibiotics; however, there were no statistically significant increases $(P$ $\geq 0.05$ ). In the presence of G6P, the anti-biofilm formation effect of VAN monotherapy was greater than that of the combination of FM and
Efa isolates. a, b 8714; c, d 13470; e, f 19663; g, h 24393; i, j 31890; k, 1 ATCC 29212

VAN. Compared with control, pairwise comparison analysis showed that DAP monotherapy $(47.1 \mathrm{mg} / \mathrm{L}) \quad$ (adjusted $P \leq 0.048)$ and $\mathrm{FM}$ $(83 \mathrm{mg} / \mathrm{L})$ combined with DAP $(20.6 \mathrm{mg} / \mathrm{L})(\mathrm{ad}-$ justed $P \leq 0.045$ ) could inhibit the biofilm formation. The anti-biofilm formation effect of FM $(83 \mathrm{mg} / \mathrm{L})$ combined with DAP $(20.6 \mathrm{mg} / \mathrm{L})$ was 

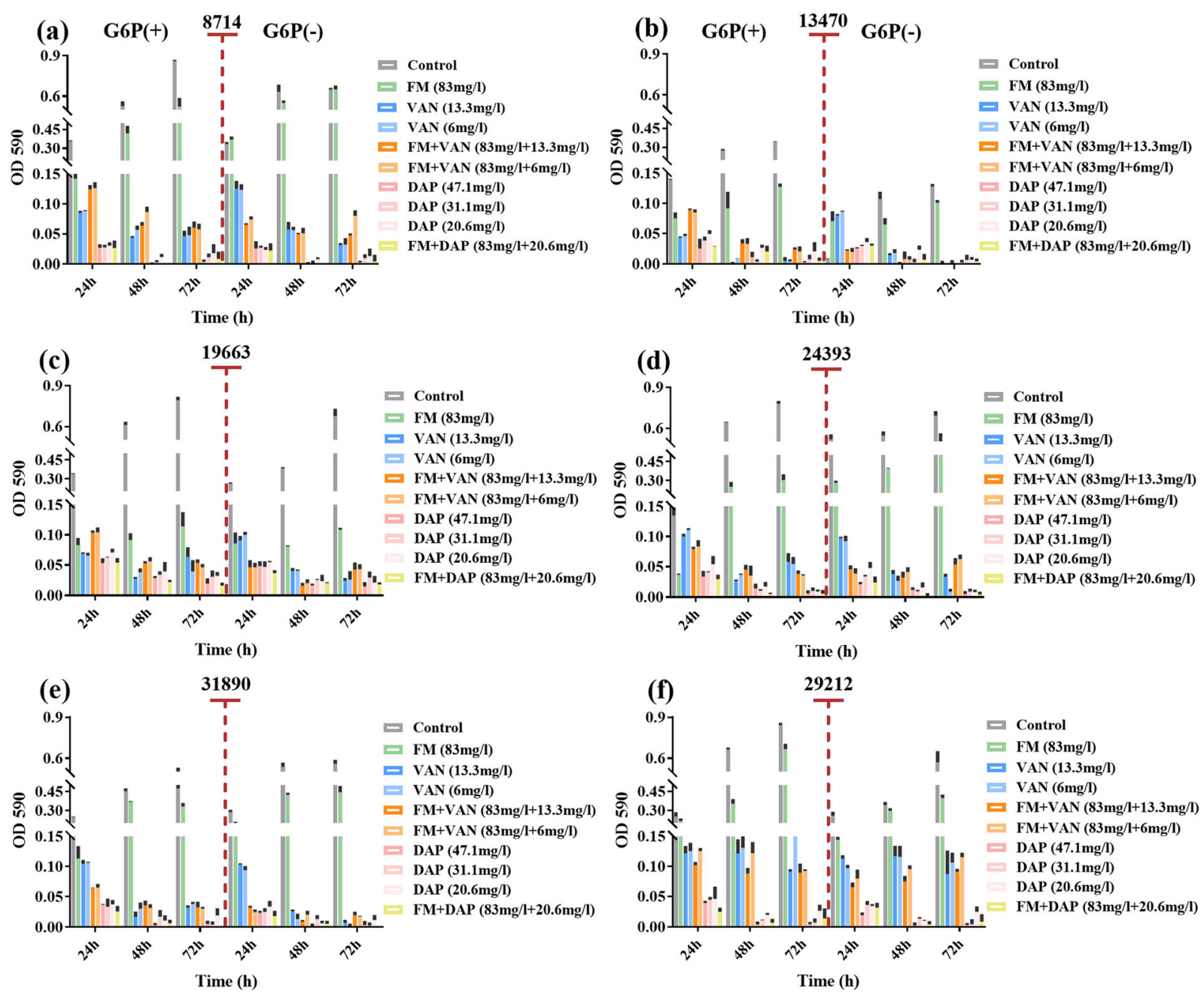

Fig. 3 Anti-biofilm formation activity of fosfomycin (FM), vancomycin (VAN) and daptomycin (DAP) monotherapy and in combination against ATCC 29212 and five LZR-Efa isolates with or without glucose-6-

similar to that of DAP monotherapy $(47.1 \mathrm{mg} /$ L).

FM monotherapy and the combination of FM and VAN with G6P showed visible biofilmeradicating effects against biofilms grown for $24 \mathrm{~h}$. G6P increased the biofilm-eradicating effect of FM combined with VAN. Interestingly, DAP alone and combined with FM showed the best biofilm eradication effect against established biofilms grown for $72 \mathrm{~h}$ (Supplementary Fig. 1).

phosphate (G6P). a 8714; b 13470; c 19663; d 24393; e 31890; f ATCC 29212

\section{DISCUSSION}

Currently, E. faecium and E. faecalis are recognized as the major nosocomial human pathogens worldwide [14]. Moreover, the increasing prevalence of VRE and LZR-Efa in many countries poses a constraint on therapeutic options. Only a few in vitro and in vivo studies have reported the effects of antibiotics against LZREfa. In addition, clinical data are lacking. In this study, the activities of FM, VAN and DAP, alone and in combination, against planktonic LZR-Efa 
cells and those forming biofilms were tested in vitro.

The lethal effect of DAP monotherapy was much more pronounced than for FM and VAN against planktonic and biofilm-forming LZR-Efa isolates. FM combined with VAN did not show significant synergistic effects, while FM combined with DAP showed increased bactericidal activity, a finding consistent with previous reports $[8,15]$. It is of note that the biofilm elimination effects of FM combined with VAN or DAP were different for new and mature biofilms.

FM, as an "old" antibiotic, has a broad spectrum of antimicrobial activity [16]. However, FM monotherapy allows the rapid selection of resistant variants [10]. The antibacterial mode of action of FM against LZR-Efa remains unclear. In the present study, we found the killing effect of FM against five clinical LZR-Efa isolates, and ATCC 29212 was not enhanced by increasing concentration above $1 \times$ MIC. In contrast, another study showed that FM at $16 \times$ MIC had a greater bactericidal effect $(>3 \log 10 \mathrm{CFU} / \mathrm{mL})$ at $24 \mathrm{~h}$ against $E$. faecalis (ATCC 19433) than at lower concentrations. However, no concentration-dependent activity was observed against adherent $E$. faecalis [15]. One possible explanation for these differences is that the different resistance of antibiotics among E. faecalis could influence the actions of FM.

Despite the limitations of therapeutic options for treating LZR-Efa infections, susceptibility to VAN remains high [3]. However, a synergistic interaction between FM and VAN was not observed in our experiments. In guinea pigs with foreign-body $E$. faecalis infections, the decreased bacterial count with FM alone was greater than that for FM combined with VAN [15]. Although no synergistic effect was observed, Yoshiyama et al. demonstrated that combination therapy using FM and VAN in rats could reduce the toxic effect of VAN on the kidneys [17].

Here, serum concentrations of FM combined with DAP showed synergistic activity at $24 \mathrm{~h}$ against all six isolates tested. These results are in accordance with previous in vitro studies, an in vivo infection model, and the observation that patients treated with FM and DAP have better outcomes than when used singly against methicillin-resistant Staphylococcus aureus (MRSA) and VRE [8, 15, 18, 19]. Furthermore, it was shown in the present study that the effectiveness of FM $(83 \mathrm{mg} / \mathrm{L})$ combined with DAP $(20.6 \mathrm{mg} / \mathrm{L})$ was similar to that of DAP monotherapy $(47.1 \mathrm{mg} / \mathrm{L})$ against six isolates at $24 \mathrm{~h}$. These findings indicate that the combination of FM and DAP could reduce the required dosage of $\mathrm{DAP}$, providing a benefit for patients with renal function impairment.

Studies with biofilms revealed that $E$. faecalis and MRSA are difficult to eradicate, leading to the emergence of resistance and failure of antibiotic treatment $[5,15]$. Several studies have confirmed that combinations of FM and VAN have synergistic bactericidal effects on MRSA strains embedded in biofilms [20]. Notably, the effects of FM combined with VAN or DAP tested on LZR-Efa biofilms were different. The activity of FM combined with VAN against a new biofilm was stronger than against a mature biofilm. An examination of FM combined with DAP proved the contrary. Therefore, treatments for established biofilms should be further investigated to obtain maximum effect.

There are several potential limitations. First, the biofilm formation was not captured using scanning electronic microscopy or transmission electron microscopy. Second, no in vivo studies were conducted.

\section{CONCLUSIONS}

The most efficient regimen for killing planktonic LZR-Efa cells and biofilms was a combination of FM and DAP. The addition of G6P promoted the formation of biofilm. For newly formed biofilms, FM combined with VAN showed the best biofilm elimination result, while the effect of FM combined with DAP was better for mature biofilms. Further in vivo investigation and clinical trials are needed to define the effects of different drug combinations. 


\section{ACKNOWLEDGEMENTS}

Funding. This work was supported by the Department of Health of Zhejiang province [2019312060]. The Rapid Service Fees were funded by the authors.

Authorship. All named authors meet the International Committee of Medical Journal Editors (ICMJE) criteria for authorship for this article, take responsibility for the integrity of the work as a whole, and have given their approval for this version to be published.

Disclosures. Wei Yu, Jiajie Zhang, Jiepeng Tong, Li Zhang, Yaqiong Zhan, Yicheng Huang and Yunqing Qiu have nothing to declare.

Compliance with Ethics Guidelines. This study was approved by the Ethics Committee of the First Affiliated Hospital, College of Medicine, Zhejiang University with written informed consent from all subjects (Reference Number 2018502).

Data Availability. All data generated or analyzed during this study are included in this published article/as supplementary information files.

Open Access. This article is licensed under a Creative Commons Attribution-NonCommercial 4.0 International License, which permits any non-commercial use, sharing, adaptation, distribution and reproduction in any medium or format, as long as you give appropriate credit to the original author(s) and the source, provide a link to the Creative Commons licence, and indicate if changes were made. The images or other third party material in this article are included in the article's Creative Commons licence, unless indicated otherwise in a credit line to the material. If material is not included in the article's Creative Commons licence and your intended use is not permitted by statutory regulation or exceeds the permitted use, you will need to obtain permission directly from the copyright holder. To view a copy of this licence, visit http://creativecommons.org/licenses/by$\mathrm{nc} / 4.0 /$.

\section{REFERENCES}

1. Bi R, Qin T, Fan W, Ma P, Gu B. The emerging problem of linezolid-resistant enterococci. J Glob Antimicrob Resist. 2018;13:11-9. https://doi.org/ 10.1016/j.jgar.2017.10.018.

2. Deshpande LM, Castanheira M, Flamm RK, Mendes RE. Evolving oxazolidinone resistance mechanisms in a worldwide collection of enterococcal clinical isolates: results from the SENTRY Antimicrobial Surveillance Program. J Antimicrob Chemother. 2018;73:2314-22. https://doi.org/10.1093/jac/ dky188.

3. Sadowy E. Linezolid resistance genes and genetic elements enhancing their dissemination in enterococci and streptococci. Plasmid. 2018;99:89-988. https://doi.org/10.1016/j.plasmid.2018.09.011.

4. Chen M, Pan H, Lou $\mathrm{Y}$, et al. Epidemiological characteristics and genetic structure of linezolidresistant Enterococcus faecalis. Infect Drug Resist. 2018;11:2397-409. https://doi.org/10.2147/IDR. S181339.

5. Tian Y, Li T, Zhu Y, Wang B, Zou X, Li M. Mechanisms of linezolid resistance in staphylococci and enterococci isolated from two teaching hospitals in Shanghai, China. BMC Microbiol. 2014;14:292. https://doi.org/10.1186/s12866-014-0292-5.

6. Lewis K. Riddle of biofilm resistance. Antimicrob Agents Chemother. 2001;45:999-1007. https://doi. org/10.1128/AAC.45.4.999-1007.2001.

7. Tang HJ, Chen CC, Zhang CC, et al. In vitro efficacy of fosfomycin-based combinations against clinical vancomycin-resistant enterococcus isolates. Diagn Microbiol Infect Dis. 2013;77(3):254-7. https://doi. org/10.1016/j.diagmicrobio.2013.07.012.

8. Zheng JX, Sun X, Lin ZW, et al. In vitro activities of daptomycin combined with fosfomycin or rifampin on planktonic and adherent linezolid-resistant isolates of Enterococcus faecalis. J Med Microbiol. 2019;68(3):493-502. https://doi.org/10.1099/jmm. 0.000945 .

9. Clinical and Laboratory Standards Institute. Performance standards for antimicrobial susceptibility testing. 26th informational supplement 2016. https://www.clsi.org/. Accessed Jan, 2016. 
10. $\mathrm{Yu} \mathrm{W}$, Shen $\mathrm{P}, \mathrm{Bao} \mathrm{Z}$, et al. In vitro antibacterial activity of fosfomycin combined with other antimicrobials against KPC-producing Klebsiella pneumoniae. Int J Antimicrob Agents. 2017;50(2): 237-41. https://doi.org/10.1016/j.ijantimicag.2017. 03.011 .

11. Tängdén T, Hickman RA, Forsberg P, Lagerbäck P, Giske CG, Cars O. Evaluation of double- and tripleantibiotic combinations for VIM- and NDM-producing Klebsiella pneumoniae by in vitro time-kill experiments. Antimicrob Agents Chemother. 2014;58:1757-62. https://doi.org/10.1128/AAC. 00741-13.

12. Shokouhi S, Alavi DI. Determination of vancomycin trough level in serum and cerebrospinal fluid of patients with acute community-acquired meningitis: a prospective study. J Infect. 2014;69(5):424-9. https://doi.org/10.1016/j.jinf. 2014.06.010.

13. D'Avolio A, Pensi D, Baietto L, Pacini G, Di Perri G, De Rosa FG. Daptomycin pharmacokinetics and pharmacodynamics in septic and critically ill patients. Drugs. 2016;76(12):1161-74. https://doi. org/10.1007/s40265-016-0610-3.

14. Bender JK, Cattoir V, Hegstad K, et al. Update on prevalence and mechanisms of resistance to linezolid, tigecycline and daptomycin in enterococci in Europe: towards a common nomenclature. Drug Resist Updat. 2018;40:25-39. https://doi.org/10. 1016/j.drup.2018.10.002.

15. Oliva A, Furustrand Tafin U, Maiolo EM, Jeddari S, Bétrisey B, Trampuz A. Activities of fosfomycin and rifampin on planktonic and adherent Enterococcus faecalis strains in an experimental foreign-body infection model. Antimicrob Agents Chemother. 2014;58(3):1284-93. https://doi.org/10.1128/AAC. 02583-12.

16. Falagas ME, Kastoris AC, Karageorgopoulos DE, Rafailidis PI. Fosfomycin for the treatment of infections caused by multidrug-resistant non-fermenting Gram-negative bacilli: a systematic review of microbiological, animal and clinical studies. Int J Antimicrob Agents. 2009;34:111-20.

17. Yoshiyama Y, Yazaki T, Wong PC, Beauchamp D, Kanke M. The effect of fosfomycin on glycopeptide antibiotic-induced nephrotoxicity in rats. J Infect Chemother. 2001;7(4):243-6. https://doi.org/10. $1007 / \mathrm{s} 101560170020$.

18. García-de-la-Mària C, Gasch $\mathrm{O}$, García-Gonzalez J, et al. The combination of daptomycin and fosfomycin has synergistic, potent, and rapid bactericidal activity against methicillin-resistant Staphylococcus aureus in a rabbit model of experimental endocarditis. Antimicrob Agents Chemother. 2018;62(6):e02633-e2717. https://doi.org/ 10.1128/AAC.02633-17.

19. Miró JM, Entenza JM, Del Río A, et al. High-dose daptomycin plus fosfomycin is safe and effective in treating methicillin-susceptible and methicillin-resistant Staphylococcus aureus endocarditis. Antimicrob Agents Chemother. 2012;56(8):4511-5. https://doi.org/10.1128/AAC.06449-11.

20. Shi J, Mao NF, Wang L, et al. Efficacy of combined vancomycin and fosfomycin against methicillinresistant Staphylococcus aureus in biofilms in vivo. PLoS One. 2014;9(12):e113133. https://doi.org/10. 1371/journal.pone.0113133. 